\title{
PSEUDO-PARALLEL SUBMANIFOLDS WITH FLAT NORMAL BUNDLE OF SPACE FORMS
}

\author{
G. A. LOBOS and R. TOJEIRO \\ Universidade Federal de São Carlos, 13565-905-São Carlos, Brazil \\ e-mail:lobos@dm.ufscar.br,tojeiro@dm.ufscar.br
}

(Received 7 August, 2005; accepted 4 November, 2005)

\begin{abstract}
We provide a complete local classification of pseudo-parallel submanifolds with flat normal bundle of space forms, extending the classification by Dillen-Nölker for the semi-parallel case.
\end{abstract}

2000 Mathematics Subject Classification. 53B25.

1. Introduction. Semi-parallel submanifolds of space forms have been extensively studied over the past decades. (See [11] and the references therein.) In particular, a local classification of semi-parallel submanifolds with flat normal bundle of space forms was obtained in [6]. (See also [12].) Recall that an isometric immersion $f: M^{n} \rightarrow \mathbb{Q}_{c}^{N}$ of a Riemannian manifold of dimension $n$ into a complete simply connected space form of constant sectional curvature $c$ and dimension $N$ is called semi-parallel if its second fundamental form $\alpha: T M^{n} \times T M^{n} \rightarrow T^{\perp} M^{n}$ with values in the normal bundle satisfies

$$
\bar{R}(X, Y) \cdot \alpha:=\left(\left[\bar{\nabla}_{X}, \bar{\nabla}_{Y}\right]-\bar{\nabla}_{[X, Y]}\right) \cdot \alpha=0,
$$

for all $X, Y \in T M^{n}$. In particular, this is the case if $\alpha$ is parallel, in the sense that $\bar{\nabla} \alpha=0$. Here $\bar{\nabla}$ stands for the van der Waerden-Bortolotti connection of $f$, given by

$$
\left(\bar{\nabla}_{X} \alpha\right)(V, W)=\nabla_{X}^{\perp} \alpha(V, W)-\alpha\left(\nabla_{X} V, W\right)-\alpha\left(V, \nabla_{X} W\right)
$$

where $\nabla$ and $\nabla^{\perp}$ denote the Levi-Civita connection of $M^{n}$ and the normal connection of $f$, respectively.

Semi-parallel submanifolds are, intrinsically, semi-symmetric Riemannian manifolds [14], [15]; that is, their curvature tensor $R$ satisfies

$$
R(X, Y) \cdot R:=\left(\left[\nabla_{X}, \nabla_{Y}\right]-\nabla_{[X, Y]}\right) \cdot R=0
$$

for all $X, Y \in T M^{n}$, which is the integrability condition for the equation $\nabla R=0$ that characterizes locally symmetric Riemannian manifolds.

Pseudo-parallel submanifolds were introduced in [1], [2] as natural extensions of semi-parallel submanifolds and as the extrinsic analogues of pseudo-symmetric Riemannian manifolds in the sense of Deszcz [5], which generalize semi-symmetric Riemannian manifolds. An isometric immersion $f: M^{n} \rightarrow \mathbb{Q}_{c}^{N}$ is said to be pseudoparallel if there exists a smooth function $\phi$ on $M^{n}$ such that

$$
\bar{R}(X, Y) \cdot \alpha=\phi(X \wedge Y) \cdot \alpha,
$$


for all $X, Y \in T M^{n}$, where

$$
((X \wedge Y) \cdot \alpha)(Z, W)=-\alpha((X \wedge Y) Z, W)-\alpha((X \wedge Y) W, Z),
$$

the case $\phi=0$ corresponding to semi-parallel isometric immersions.

The aim of this paper is to provide a complete local classification of pseudo-parallel submanifolds with flat normal bundle of space forms, extending the classification in [6] for the semi-parallel case.

2. The result. Given an isometric immersion $f: M^{n} \rightarrow \mathbb{Q}_{c}^{N}$ with flat normal bundle (i.e., with vanishing normal curvature tensor), it is well known (cf. [6]) that, for each point $x \in M^{n}$, there exist an integer $s=s(x) \in\{1, \ldots, n\}$ and a uniquely determined subset $H_{x}=\left\{\eta_{1}, \ldots, \eta_{s}\right\}$ of $T_{x}^{\perp} M^{n}$ such that $T_{x} M^{n}$ is the orthogonal sum of the nontrivial subspaces

$$
E_{\eta_{i}}(x)=\left\{X \in T_{x} M: \alpha(X, Y)=\langle X, Y\rangle \eta_{i}, \forall Y \in T_{x} M\right\}(1 \leq i \leq s) .
$$

Thus, the second fundamental form of $f$ has the simple representation

$$
\alpha(X, Y)=\sum_{i=1}^{s}\left\langle X_{i}, Y_{i}\right\rangle \eta_{i},
$$

or equivalently, for any $\xi \in T_{x}^{\perp} M^{n}$ the shape operator $A_{\xi}$ satisfies

$$
A_{\xi} X=\sum_{i=1}^{s}\left\langle\xi, \eta_{i}\right\rangle X_{i}
$$

where $X \mapsto X_{i}$ denotes orthogonal projection onto $E_{\eta_{i}}(x)$. Each $\eta_{i} \in H_{x}$ is called a principal normal vector of $f$ at $x$, and the rank of $E_{\eta_{i}}(x)$ is the multiplicity of $\eta_{i}$. The Gauss equation takes the form

$$
R(X, Y)=\sum_{i, j=1}^{s}\left(\left\langle\eta_{i}, \eta_{j}\right\rangle+c\right) X_{i} \wedge Y_{j},
$$

where $R$ denotes the curvature tensor of $M^{n}$ and, for $X, Y \in T M^{n}, X \wedge Y$ stands for the endomorphism of $T M^{n}$ given by

$$
(X \wedge Y) Z=\langle Z, Y\rangle X-\langle Z, X\rangle Y .
$$

Whenever the function $M^{n} \rightarrow\{1, \ldots, n\}$ given by $x \mapsto \# H_{x}$ (number of elements of $H_{x}$ ) has a constant value $s$ on an open subset $U \subset M^{n}$, there are smooth normal vector fields $\eta_{1}, \ldots, \eta_{s}$ along $U$, called the principal normal vector fields of $f$ on $U$, such that $H_{x}=\left\{\eta_{1}(x), \ldots, \eta_{s}(x)\right\}$ for any $x \in U$. Moreover, $E_{\eta_{i}}=\left(E_{\eta_{i}}(x)\right)_{x \in U}$ is a $C^{\infty}$ subbundle of $T U$ for $i=1, \ldots, s$. of [6].)

The following facts are well known. (See, e.g., Lemma 2.2-(b) and equation 2.5

LeMma 2.1. Let $f: M^{n} \rightarrow \mathbb{R}^{N}$ be an isometric immersion with flat normal bundle. Assume that the number of distinct principal normal vectors of $f$ at any point is constant on $M^{n}$.

(i) Each principal normal vector field $\eta_{i}$ is parallel in the normal connection $\nabla^{\perp}$ along $E_{\eta_{i}}$ whenever the rank of $E_{\eta_{i}}$ is at least two. 
(ii) For any local sections $X_{i}$ of $E_{\eta_{i}}$ and $X_{j}, Y_{j}$ of $E_{\eta_{j}}$, $i \neq j$, we have

$$
\left\langle\nabla_{X_{j}} Y_{j}, X_{i}\right\rangle\left(\eta_{j}-\eta_{i}\right)=\left\langle X_{j}, Y_{j}\right\rangle \nabla_{X_{i}}^{\perp} \eta_{j}
$$

The condition for an isometric immersion to be pseudo-parallel takes the following simple form when it has flat normal bundle.

LEMMA 2.2. Let $f: M^{n} \rightarrow \mathbb{Q}_{c}^{N}$ be an isometric immersion with flat normal bundle and let $\phi \in C^{\infty}\left(M^{n}\right)$. Then $f$ is $\phi$-pseudo parallel if and only if, for every $x \in M^{n}$, the distinct principal normal vectors $\eta_{1}, \ldots, \eta_{s}$ off at $x$ satisfy

$$
\left\langle\eta_{i}, \eta_{j}\right\rangle+c=\phi(x), \quad 1 \leq i \neq j \leq s .
$$

Proof. A straightforward computation shows that an isometric immersion $f: M^{n} \rightarrow \mathbb{Q}_{c}^{N}$ is $\phi$-pseudo-parallel if and only if

$$
\begin{aligned}
R^{\perp}(X, Y) \alpha(Z, W)= & \alpha(R(X, Y) Z, W)+\alpha(Z, R(X, Y) W) \\
& -\phi\langle Y, Z\rangle \alpha(X, W)+\phi\langle X, Z\rangle \alpha(Y, W) \\
& -\phi\langle Y, W\rangle \alpha(Z, X)+\phi\langle X, W\rangle \alpha(Z, Y)
\end{aligned}
$$

for all $X, Y, Z, W \in T M^{n}$, where $R^{\perp}$ denotes the normal curvature tensor of $f$. When $R^{\perp}$ vanishes identically it is easily checked using (2.1) and (2.3) that (2.6) reduces to $(2.5)$.

As a first consequence of Lemma 2.2 we have the following result.

Corollary 2.3. Any isometric immersion $f: M^{n} \rightarrow \mathbb{Q}_{c}^{N}$ with flat normal bundle that has exactly two distinct principal normal vector fields $\eta_{1}, \eta_{2}$ is $\phi$-pseudo parallel with $\phi=\left\langle\eta_{1}, \eta_{2}\right\rangle+c$.

In view of Corollary 2.3, a first step towards the classification of pseudo-parallel submanifolds with flat normal bundle is to determine the submanifolds with flat normal bundle that have exactly two distinct principal normal vector fields. With that goal of independent interest in mind, we first observe that if the principal normal vector fields of an isometric immersion $f: M^{n} \rightarrow \mathbb{Q}_{c}^{N}$ with flat normal bundle span a onedimensional subbundle $N_{1}^{f}$ of the normal bundle, then it follows from (2.4) that $N_{1}^{f}$ is parallel in the normal connection, unless there are exactly two distinct principal normal vector fields and one of them is zero and has multiplicity $n-1$. Thus, either $f\left(M^{n}\right)$ is contained in a totally geodesic submanifold $\mathbb{Q}_{c}^{n+1} \subset \mathbb{Q}_{c}^{N}$ or $M^{n}$ has constant sectional curvature $c$ (cf. [9, Theorem 1]). Therefore, in the next two propositions we assume that the two distinct principal normal vector fields are everywhere linearly independent.

Recall that a hypersurface $f: M^{n} \rightarrow \mathbb{Q}_{c}^{n+1}$ is a cyclide of Dupin if it has exactly two distinct eigenvalues everywhere, both of which are constant along the corresponding eigenbundles. We refer to [4] for the classification of the cyclides of Dupin. The next result is contained in Proposition 24 of [10], but a direct proof is included here for the convenience of the reader.

Proposition 2.4. Let $f: M^{n} \rightarrow \mathbb{Q}_{c}^{N}$ be an isometric immersion with flat normal bundle. Assume that $f$ has exactly two distinct principal normal vector fields, which are linearly independent and parallel along the corresponding eigenbundles. Then $f=i \circ g$, where $g: M \rightarrow \mathbb{Q}_{\tilde{c}}^{n+1}$ is a cyclide of Dupin and $i: \mathbb{Q}_{\tilde{c}}^{n+1} \rightarrow \mathbb{Q}_{c}^{N}$ is an umbilical inclusion. 
Proof. Equation (2.4) and the assumption that the principal normal vector field $\eta_{i}$ is parallel along $E_{\eta_{i}}$, for $i=1,2$, imply that the first normal bundle $N_{1}^{f}$ of $f$, i.e., the normal subbundle spanned by $\eta_{1}$ and $\eta_{2}$, is parallel in the normal connection. Let $\xi$ be a unit vector field in $N_{1}^{f}$ orthogonal to $\eta_{1}-\eta_{2}$. Then $\xi$ is an umbilical normal vector field by (2.2). Moreover, for $1 \leq i \neq j \leq 2$ we have from (2.4) that

$$
\begin{aligned}
0 & =\left\langle\nabla_{X_{i}}^{\perp} \eta_{j}, \xi\right\rangle=X_{i}\left\langle\eta_{j}, \xi\right\rangle-\left\langle\eta_{j}, \nabla_{X_{i}}^{\perp} \xi\right\rangle=X_{i}\left\langle\eta_{i}, \xi\right\rangle-\left\langle\eta_{j}, \nabla_{X_{i}}^{\perp} \xi\right\rangle \\
& =\left\langle\eta_{i}-\eta_{j}, \nabla_{X_{i}}^{\perp} \xi\right\rangle .
\end{aligned}
$$

Thus $\nabla_{X_{i}}^{\perp} \xi \in N_{1}^{f}$ is orthogonal to $\eta_{1}-\eta_{2}$ and so must vanish. Therefore $\xi$ is parallel in the normal connection, and we conclude that $f\left(M^{n}\right)$ is contained in an umbilical submanifold $\mathbb{Q}_{\tilde{c}}^{n+1} \subset \mathbb{Q}_{c}^{N}$.

A hypersurface $f: M^{n} \rightarrow \mathbb{Q}_{c}^{n+1}$ is said to be quasi-umbilic if it has everywhere a principal curvature of multiplicity at least $n-1$. Any quasi-umbilic hypersurface is conformally flat, and the converse is also true for $n \geq 4$. Quasi-umbilic hypersurfaces $f: M^{n} \rightarrow \mathbb{Q}_{c}^{n+1}$ with no umbilic points carry a codimension one foliation by umbilical leaves in both $M^{n}$ and $\mathbb{Q}_{c}^{n+1}$. (See [3] for a parametric description of such hypersurfaces.)

The next proposition completes, in view of part (i) of Lemma 2.1, the classification of isometric immersions with flat normal bundle that have exactly two distinct principal normal vector fields.

Proposition 2.5. Let $f: M^{n} \rightarrow \mathbb{Q}_{c}^{N}, n \geq 3$, be an isometric immersion with flat normal bundle. Assume that $f$ has exactly two linearly independent principal normal vector fields $\eta_{1}, \eta_{2}$ and that rank $E_{\eta_{1}}=1$. Then $f=h \circ g$, where $g: M^{n} \rightarrow \mathbb{Q}_{c}^{n+1}$ is a quasi-umbilic hypersurface and $h: U \subset \mathbb{Q}_{c}^{n+1} \rightarrow \mathbb{Q}_{c}^{N}$ is an isometric immersion with no totally geodesic points of an open subset $U \supset g\left(M^{n}\right)$. Moreover, on the open set of nonumbilic points of $g$ each leaf of the umbilical foliation of $g$ is mapped into a relative nullity leaf of $h$. Conversely, any such isometric immersion $f=h \circ g$, with $g$ free of umbilic points, has flat normal bundle and exactly two linearly independent principal normal vector fields, one of which is of multiplicity one.

Proof. We first prove the converse. The second fundamental form of $f$ is given by

$$
\alpha_{f}(X, Y)=h_{*} \alpha_{g}(X, Y)+\alpha_{h}\left(g_{*} X, g_{*} Y\right),
$$

for all $X, Y \in T M^{n}$. Let $\xi$ be a unit vector field normal to $g$ and let $Z$ be a unit vector field that spans the orthogonal complement $E_{\lambda}^{\perp}$ in $T M^{n}$ of the umbilical distribution $E_{\lambda}$ of $g$ correspondent to its principal curvature $\lambda$ of multiplicity $n-1$. By the assumption that the relative nullity leaves of $h$ contain the images by $g$ of the leaves of $E_{\lambda}$, we have

$$
\alpha_{f}(X, Y)=\left\langle X_{1}, Y_{1}\right\rangle \eta_{1}+\left\langle X_{2}, Y_{2}\right\rangle \eta_{2}
$$

where $\eta_{1}=\alpha_{f}(Z, Z), \eta_{2}=\lambda h_{*} \xi$ and $X \mapsto X_{1}$ (resp., $X \mapsto X_{2}$ ) denotes orthogonal projection onto $E_{\lambda}^{\perp}$ (resp., $\left.E_{\lambda}\right)$. Since $\eta_{1}=\mu h_{*} \xi+\alpha_{h}\left(g_{*} Z, g_{*} Z\right)$, where $\mu$ is the principal curvature of $g$ of multiplicity one, it follows that $\eta_{1}, \eta_{2}$ are linearly independent everywhere, because $h$ has no totally geodesic points. Moreover, $\eta_{1}$ has multiplicity one, for $Z$ spans $E_{\eta_{1}}$.

Now we prove the direct statement. Let $L$ be the line subbundle of $T^{\perp} M^{n}$ spanned by $\eta_{2}$. Denote by $\gamma$ the component of $\alpha$ in $L^{\perp}$, the orthogonal complement of $L$ in $T^{\perp} M^{n}$. It follows from (2.2) that $\operatorname{ker}(\gamma)$, the kernel of $\gamma$, coincides with $E_{\eta_{2}}$. In 
particular, since $E_{\eta_{2}}$ has rank $n-1$, this implies that the component of $\alpha$ in $L$ satisfies the Gauss equation for an isometric immersion of $M^{n}$ into $\mathbb{Q}_{c}^{n+1}$. We claim that it also satisfies the Codazzi equation. In fact, setting $\zeta=\eta_{2} /\left\|\eta_{2}\right\|$, this is equivalent to

$$
A_{\nabla_{X} \zeta} Y=A_{\nabla_{Y}} X, \text { for all } X, Y \in T M^{n},
$$

which follows from the fact that $\zeta$ is parallel along $E_{\eta_{2}}=\operatorname{ker}(\gamma)$, as follows from Lemma 2.1-(i), and $\nabla_{X}^{\perp} \zeta \in L^{\perp}$ for all $X \in T M^{n}$.

We obtain from Theorem 5 in [7] that $f=h \circ g$, where $g: M^{n} \rightarrow \mathbb{Q}_{c}^{n+1}$ is an isometric immersion with shape operator $A_{\zeta}$ and $h: U \subset \mathbb{Q}_{c}^{n+1} \rightarrow \mathbb{Q}_{c}^{N}$ is an isometric immersion of an open subset $U \supset g\left(M^{n}\right)$. It follows from (2.2) that $g$ has principal curvatures $\left\langle\zeta, \eta_{1}\right\rangle$ and $\left\langle\zeta, \eta_{2}\right\rangle$, whence it is quasi-umbilic. Since $\eta_{1}$ and $\eta_{2}$ are everywhere linearly independent, $h$ can have no totally geodesic points. Finally, that the relative nullity leaves of $h$ contain the leaves of the umbilical foliation of $g$ on the subset of nonumbilic points of $g$ follows from the proof of Theorem 5 in [7].

The study of pseudo parallel submanifolds with flat normal bundle having more than two distinct principal normal vector fields is simplified by the next result.

LemMa 2.6. Let $f: M^{n} \rightarrow \mathbb{Q}_{c}^{N}$ be a $\phi$-pseudo parallel isometric immersion with flat normal bundle of a connected Riemannian manifold. Assume that the number of distinct principal normal vectors $\eta_{1}, \ldots, \eta_{s}$ of $f$ at any point is a constant $s \geq 3$. Then $\phi$ is constant.

Proof. Taking the inner product of both sides of (2.4) with $\eta_{k}, k \neq i, j$, it follows, using Lemma 2.2, that

$$
\left\langle\nabla_{X_{i}}^{\perp} \eta_{j}, \eta_{k}\right\rangle=0, \quad i \neq j \neq k \neq i .
$$

Therefore, for $i=1, \ldots, s$, taking $j, k \neq i$ with $1 \leq j \neq k \leq s$ yields

$$
X_{i}(\phi)=X_{i}\left\langle\eta_{j}, \eta_{k}\right\rangle=\left\langle\nabla_{X_{i}}^{\perp} \eta_{j}, \eta_{k}\right\rangle+\left\langle\eta_{j}, \nabla_{X_{i}}^{\perp} \eta_{k}\right\rangle=0 .
$$

Recall that all warped product representations of space forms, that is, isometries of warped products of Riemannian manifolds onto open subsets of space forms, were classified in [13]. They are essentially restrictions of explicitly constructible isometries $\psi: N_{0} \times_{\sigma_{1}} N_{1} \times_{\sigma_{2}} \cdots \times_{\sigma_{l}} N_{\ell} \rightarrow \mathbb{Q}_{c}^{N}$ onto open dense subsets of $\mathbb{Q}_{c}^{N}$, where $N_{1}, \ldots, N_{\ell}$ are complete spherical submanifolds of $\mathbb{Q}_{c}^{N}$ through a common point $\bar{p} \in \mathbb{Q}_{c}^{N}$, whose mean curvature vectors $a_{1}, \ldots, a_{\ell}$ at $\bar{p}$ in the flat ambient space $\mathbb{O}_{0} \supset \mathbb{Q}_{c}^{N}$ are pairwise orthogonal, and $N_{0}$ is an open subset of a totally geodesic submanifold of $\mathbb{Q}_{c}^{N}$ through $\bar{p}$ whose tangent space at $\bar{p}$ contains $a_{1}, \ldots, a_{\ell}$. We refer to [13] for details. Given an isometric immersion $f_{0}: V \rightarrow N_{0}$, the map $f: V \times{ }_{\rho_{1}} N_{1} \times{ }_{\rho_{2}} \cdots \times \times_{\rho_{l}} N_{\ell} \rightarrow \mathbb{Q}_{c}^{N}$ defined by $f\left(x_{0}, \ldots, x_{\ell}\right)=\psi\left(f_{0}\left(x_{0}\right), x_{1}, \ldots, x_{\ell}\right)$, where $\rho_{i}=\sigma_{i} \circ f_{0}: V \rightarrow \mathbb{R}_{+}, \quad 1 \leq i \leq \ell$, is also an isometric immersion, called in [6] the multi-rotational submanifold with profile $f_{0}$ determined by $\psi$.

According to [6], [8], an isometric immersion $f: U \subset \mathbb{Q}_{k}^{m} \rightarrow \mathbb{O}_{0}^{N}$ into either Euclidean or Lorentzian space is said to satisfy the $k$-helix property with respect to the orthogonal vectors $w_{1}, \ldots, w_{\ell} \in \mathbb{O}_{0}^{N}$ if the height functions $h_{w_{j}}(x)=\left\langle f(x), w_{j}\right\rangle$ are the restrictions to $U$ of linear functions in the flat space $\mathbb{O}_{0} \supset \mathbb{Q}_{k}^{m}$ with pairwise orthogonal gradient vector fields, which for $k \neq 0$ are nonzero and have vanishing independent term.

We are now in a position to state and prove our main result. 
THEOREM 2.7. Let $f: M^{n} \rightarrow \mathbb{Q}_{c}^{N}$ be a $\phi$-pseudo parallel isometric immersion with flat normal bundle. Then either $n=2$ and $\phi=K$ on the open subset of non-umbilic points off, where $K$ is the Gaussian curvature of $M^{2}$, or there exists an open dense subset $\tilde{M}$ of $M^{n}$, where one of the following holds locally:

(i) $\left.f\right|_{\tilde{M}}$ is umbilical;

(ii) $\left.f\right|_{\tilde{M}}=i \circ g$, where $g: \tilde{M} \rightarrow \mathbb{Q}_{\tilde{c}}^{n+1}$ is a cyclide of Dupin and $i: \mathbb{Q}_{\tilde{c}}^{n+1} \rightarrow \mathbb{Q}_{c}^{N}$ is either an umbilical or a totally geodesic inclusion;

(iii) $\left.f\right|_{\tilde{M}}=h \circ g$, where $g: \tilde{M} \rightarrow \mathbb{Q}_{c}^{n+1}$ is a quasi-umbilic hypersurface and $h: U \subset$ $\mathbb{Q}_{c}^{n+1} \rightarrow \mathbb{Q}_{c}^{N}$ is an isometric immersion of an open subset $U \supset g(\tilde{M})$, as in Proposition 2.5 ;

(iv) $\phi=k \in \mathbb{R}$ and $\tilde{M}$ has constant sectional curvature $k$;

(v) $\phi=0$ and $\left.f\right|_{\tilde{M}}$ is an extrinsic product of spherical submanifolds of $\mathbb{Q}_{c}^{N}$;

(vi) $\phi=k \in \mathbb{R}$ and $\left.f\right|_{\tilde{M}}$ is the restriction of a multi-rotational submanifold

$$
V \times_{\rho_{1}} N_{1} \times_{\rho_{2}} \cdots \times_{\rho_{l}} N_{l} \rightarrow \mathbb{Q}_{c}^{N} \approx N_{0} \times_{\sigma_{1}} N_{1} \times_{\sigma_{2}} \cdots \times_{\sigma_{l}} N_{l}
$$

where $V \subset \mathbb{Q}_{k}^{m}, m=n-\sum_{i=1}^{l} \operatorname{dim} N_{i} \geq 1$, and the profile $f_{0}: V \rightarrow N_{0}$ is an isometric immersion with flat normal bundle satisfying the $k$-helix property with respect to the mean curvature vectors $a_{1}, \ldots$, al of $N_{1}, \ldots, N_{l}$ in the flat space $\mathbb{O}_{0} \supset \mathbb{Q}_{c}^{N}$.

Proof. If $n=2$ then, on the open subset of non-umbilic points of $f$, there are exactly two distinct principal normal vector fields $\eta_{1}$ and $\eta_{2}$, with $\left\langle\eta_{1}, \eta_{2}\right\rangle+c=K$, and the result follows from Corollary 2.3. From now on we assume that $n \geq 3$. Let $U_{1} \subset M^{n}$ be the interior of the subset where $f$ has only one principal normal vector. Then $\left.f\right|_{U_{1}}$ is umbilical. Now let $U_{2} \subset M^{n}$ be the interior of the subset where $f$ has exactly two distinct principal normal vectors. By Lemma 2.1-(i), Propositions 2.4 and 2.5 , and the comments after Corollary 2.3 , we have that $\left.f\right|_{U_{2}}$ is locally as in either of cases (ii), (iii) or (iv) (the latter occurring for $k=c$ on open subsets where the principal normal vector fields span a one-dimensional nonparallel normal subbundle). Finally, let $V \subset M^{n}$ be the open subset where the number of distinct principal normal vectors of $f$ is at least 3. By Lemma 2.6 we have that $\phi$ is constant on each connected open subset of $V$ where the number of principal normal vectors is constant. Thus, on any such subset $f$ is an isometric immersion whose principal normal vector fields have constant inner products in the sense of [8] (the constant being $\phi-c$ ). Therefore, by Theorem 24 of [8] there exists an open dense subset $U_{3}$ of $V$ such that $\left.f\right|_{U_{3}}$ is locally as in cases (iv), (v) or (vi). We conclude that the statement holds on the open dense subset $\tilde{M}=\bigcup_{i=1}^{3} U_{i}$.

REMARKS 2.8. (1) Let us discuss when an isometric immersion $f$, as in either of the cases in Theorem 2.7, is semi-parallel. First, a surface with flat normal bundle and no umbilic points is semi-parallel if and only if it is flat. An umbilical submanifold of arbitrary dimension is $\phi$-pseudo-parallel for any smooth function $\phi$, in particular it is also semi-parallel. Now, $f$ as in case (ii) is semi-parallel if and only if $\tilde{c} \neq 0$ and $g$ is an extrinsic product of two spherical submanifolds of $\mathbb{Q}_{\tilde{c}}^{n+1}$, or $\tilde{c}=0$ and $g$ is an extrinsic product of either a round sphere or a round cone and a linear subspace. In case (iii), $f$ is semi-parallel if and only if $h$ is totally geodesic and $g$ is a rotational hypersurface over a curve in a two-dimensional totally geodesic geodesic submanifold $\mathbb{Q}_{c}^{2}$ of $\mathbb{Q}_{c}^{n+1}$, which is either a straight line in $\mathbb{R}^{2}$ if $c=0$ (in which case $f$ is a round cone) or a helix in the underlying three-dimensional flat space $\mathbb{O}_{0}^{3} \supset \mathbb{Q}_{c}^{2}$; i.e., either Euclidean space $\mathbb{R}^{3}$ or Lorentzian space $\mathbb{L}^{3}$, according as $c>0$ or $c<0$, respectively. Clearly, $f$ as in (iv) is 
semi-parallel if and only if $k=0$; that is, $M^{n}$ is flat, whereas $f$ is always semi-parallel (in fact parallel) in case (v). Finally, in (vi) the semi-parallel case occurs when $k=0$; that is, when $f$ is a multi-rotational submanifold whose profile is a flat submanifold with flat normal bundle satisfying the helix property.

(2) It was proved in [1] that a $\phi$-pseudo-parallel isometric immersion $f: M^{n} \rightarrow$ $\mathbb{Q}_{c}^{n+2}$ has automatically flat normal bundle at any point where its mean curvature vector $H$ does not vanish. Moreover, if $H(p)=0$ and $\phi(p) \geq c$, then $f$ is totally geodesic at $p$.

\section{REFERENCES}

1. A. C. Asperti, G. A. Lobos and F. Mercuri, Pseudo-parallel immersions in space forms, Mat. Contemp. 17 (1999), 59-70.

2. A. C. Asperti, G. A. Lobos and F. Mercuri, Pseudo-parallel submanifolds of a space form, Adv. Geom. 2 (2002), 57-71.

3. M. do Carmo, M. Dajczer and F. Mercuri, Compact conformally flat hypersurfaces, Trans. Amer. Math. Soc. 288 (1985), 189-203.

4. T. Cecil, Lie sphere geometry (Springer Verlag, 1992).

5. R. Deszcz, On pseudosymmetric space, Bull. Belg. Math. Soc. 44 (1992), 1-34.

6. F. Dillen and S. Nölker, Semi-parallel submanifolds, multi-rotation surfaces and the helix-property, J. Reine Angew. Math. 435 (1993), 33-63.

7. M. Dajczer and R. Tojeiro, On compositions of isometric immersions, J. Diff. Geometry 36 (1992), 1-18.

8. M. Dajczer and R. Tojeiro, Isometric immersions and the generalized Laplace and elliptic sinh-Gordon equations, J. Reine Angew. Math. 467 (1995), 109-147.

9. M. Dajczer and R. Tojeiro, Submanifolds with nonparallel first normal bundle, Canad. Math. Bull. 37 (1995), 330-337.

10. M. Dajczer, L. Florit and R. Tojeiro, Reducibility of Dupin submanifolds, Illinois $J$. Math., to appear.

11. Ü. Lumiste, Submanifolds with parallel fundamental form, in Handbook of differential geometry, Vol. I (North-Holland, Amsterdam, 2000), 779-864.

12. Ü. Lumiste, Normally flat semi-symmetric submanifolds, in Differential geometry and its applications, Proc. Conf. Dubrovnik 1988 (Univ. Novi Sad, Novi Sad, 1989), 159-171.

13. S. Nölker, Isometric immersions of warped products, Diff. Geom. Appl. 6 (1996), 1-30.

14. Z. J. Szabó, Structure theorems on Riemannian spaces satisfying $R(X, Y) \cdot R=0$, I, The local version, J. Diff. Geom. 17 (1982), 531-582.

15. Z. J. Szabó, Structure theorems on Riemannian spaces satisfying $R(X, Y) \cdot R=0$, II, The global version, Geom. Dedicata 19 (1985), 321-348. 